\title{
Transparent organic light-emitting diodes with different bi-directional emission colors using color-conversion capping layers
}

\author{
Jonghee Lee ${ }^{1,2^{*},}$, Tae-Wook Koh ${ }^{3, \dagger}$, Hyunsu Cho ${ }^{1,3}$, Tobias Schwab ${ }^{2}$, Jae-Hyun Lee ${ }^{4}$, Simone \\ Hofmann $^{2}$, Jeong-Ik Lee ${ }^{1}$, Seunghyup Yoo ${ }^{3}$, Karl Leo ${ }^{2}$, and Malte C. Gather ${ }^{2,5}$ \\ ${ }^{1}$ OLED Research Center, Electronics and Telecommunications Research Institute (ETRI), \\ Daejeon 305-700, Republic of Korea \\ ${ }^{2}$ Institut für Angewandte Photophysik, Technische Universität Dresden, George-Bähr-Straße 1, \\ 01062 Dresden, Germany \\ ${ }^{3}$ Department of Electrical Engineering, Korea Advanced Institute of Science and Technology \\ (KAIST), Daejeon, 305-701, Republic of Korea \\ ${ }^{4}$ Department School of Global Convergence Studies, Hanbat National University, San 16-1, \\ Duckmyoung-dong, Daejeon, 305-719, Republic of Korea \\ ${ }^{5}$ SUPA, School of Physics and Astronomy, University of St Andrews, St Andrews KY16 9SS, \\ $U K$
}

\begin{abstract}
We report a study on transparent organic light-emitting diodes (OLEDs) with different bidirectional emission colors, enabled by color-conversion organic capping layers. Starting from a transparent blue OLED with an uncapped $\mathrm{Ag}$ top electrode exhibiting an average transmittance of 33.9\%, a 4-(Dicyanomethylene)-2-methyl- 6-(4-dimethylaminostyryl)-4Hpyran (DCM)-doped tris-(8-hydroxy-quinolinato)-aluminium (Alq3) capping layer is applied to achieve color-conversion from blue to orange-red on the top side while maintaining almost unchanged device transmittance. This color-conversion capping layer does not only change the color of the top side emission, but also enhances the overall device efficiency due to the optical interaction of the capping layer with the primary blue transparent OLED. Top white emission from the transparent bi-directional OLED exhibits a correlated color temperature around $6,000 \mathrm{~K}-7,000 \mathrm{~K}$, with excellent color stability as evidenced by an extremely small
\end{abstract}


variation in color coordinate of $\Delta(x, y)=(0.002,0.002)$ in the forward luminance range of $100-1000 \mathrm{~cd} \mathrm{~m}^{-2}$. At the same time, the blue emission color of bottom side is not influenced by the color conversion capping layer, which finally results in different emission colors of the two opposite sides of our transparent OLEDs.

Keywords: Down-conversion white OLED, color conversion capping layer, transparent OLED, bidirectional OLED

$\dagger$ Authors contributed equally to this work.

*Author to whom correspondence should be addressed.

*Dr. Jonghee Lee

Tel.: +82-42-860-0871; Fax: +82-42-860-5202; E-mail address: jonghee.lee@etri.re.kr

\section{Introduction}

Organic light-emitting diodes (OLEDs) are well known for offering an outstanding variety in device form factor: they can be built on plastic films or metal foils and thus can be ultrathin and flexible [1-4]; they can be designed to emit light in a broad range of colors [5-7]; and most noticeably they can be made transparent by using transparent electrodes for both anode and cathode [8-10]. This transparency feature is considered to be among the key factors that distinguish OLEDs from other light-emitting devices. Since OLEDs contain self-emissive organic emitters and the generated light can pass through transparent electrodes on either side, transparent OLEDs (TOLEDs) exhibit bi-directional emission. This also means that the emission color occurs in both top and bottom direction is almost identical in TOLEDs, although slight difference in electroluminescence spectra may exist between the emission directions due to differences in transmittance/reflectance at each electrode. 
In this work, we propose a novel type of TOLED that emits different colors in the two directions by combining a blue-emitting TOLED and an organic color-converting capping layer (CCL). We believe that this asymmetric emission color combined with the optical transparency is likely to draw noticeable attention for artistic and decorative lighting applications. Although the concept of using an organic CCL for down-conversion from blue photons to longer wavelength photons is not new, this approach has been applied only for opaque OLEDs with uni-directional emission [11-13]. Furthermore, most down-conversion type white OLEDs incorporating organic CCLs exhibit lower device efficiencies than pristine, unconverted OLEDs. In the present study, we focus on addressing the issues mentioned above by integrating the color-converting organic layer with a blue-emitting TOLED, and hence realize a TOLED with different bi-directional emission colors; while the bottom side emission remains blue, top side emission is changed to white by a partial down-conversion process. The organic CCL in our TOLED concurrently serves three purposes: (i) it works as a color-conversion layer, changing some of the primary blue photons to orange-red photons that are later mixed up to be white; (ii) it serves as optical capping layer adjusting the optical behavior of the TOLED to enhance its efficiency; (iii) it forms an outermost dielectric layer that preserves the overall visual transparency of the TOLEDs. These goals are simultaneously achieved by choosing an appropriate color-conversion organic capping layer with a suitable thickness that provides an effective conversion path length and is adequate for high transparency and enhanced efficiency.

\section{Experimental}

A series of phosphorescent TOLEDs based on $p-i-n$ doped structures with thickness variation of the CCL was fabricated using the following device architecture: indium tin oxide

(ITO) $\quad(90 \mathrm{~nm}) / p$-type layer/1,1-bis[(di-4-tolylamino)phenyl $]$ cyclohexane (TAPC) (10 $\mathrm{nm}$ )/emissive layer (EML, $10 \mathrm{~nm}$ )/2-(diphenylphosphoryl)spirofluorene (SPPO1) $(10 \mathrm{~nm}) / n$ - 
type layer/Ag(15 nm)/CCL (variable thickness, denoted as $\mathrm{X} \mathrm{nm}$ ). All samples were deposited by thermal evaporation onto ITO-coated glass substrates in a UHV chamber (Kurt J. Lesker Co.) at a base pressure of around $10^{-7} \mathrm{mbar}$. For the $p$-type hole injection / transport layer, we used a $35 \mathrm{~nm}$ thick film of $N, N, N, N$ '-tetrakis(4-methoxyphenyl)-benzidine (MeOTPD) doped with 2 wt \% of the Novaled dopant $p$-type 2 (NDP-2, Novaled AG, Dresden). For the $n$-type electron injection and transport layer, we used $30 \mathrm{~nm}$ of cesium (Cs)-doped BPhen, with conductivity comparable to the conductivity of the $p$-layer. $10 \mathrm{~nm}$ of TAPC and $10 \mathrm{~nm}$ of SPPO1 were used as electron and hole blocking layers, respectively, to confine charge carriers and excitons within the EML.[14]

The 10-nm thick blue EML consists of two host materials 4,4',4''-tris ( $N$-carbazolyl)triphenylamine (TCTA) (5 nm) and 2,6-bis(3-(carbazol-9-yl)phenyl)pyridine (26DCzPPy) (5 $\mathrm{nm}$ ), both doped with $20 \%$ of the phosphorescent emitter iridium(III) bis(4,6-(difluorophenyl) pyridinato-N,C2') picolinate (FIrpic). Finally, as CCLs, we used tris-(8-hydroxyquinolinato)-aluminium (Alq3) doped with 2 wt\% of 4-(Dicyanomethylene)-2-methyl-6-(4dimethylaminostyryl)-4H-pyran (DCM) with thicknesses varying from $0 \mathrm{~nm}$ to $265 \mathrm{~nm}$ and $435 \mathrm{~nm}$.

For TOLEDs characterization, we utilized a Keithley Source Measure Unit 2400 to obtain current-voltage data, while an INSTRUMENT SYSTEMS GmbH CAS140 spectrometer and a calibrated silicon photodiode (Hamamatsu) were used to measure luminance and spectral radiant intensity in forward direction at the same time. Afterwards, spatial emission properties of the TOLEDs were evaluated with a spectrogoniometer setup to calculate external quantum efficiencies (error ranges of the current density $(J)$ - voltage $(V)$ luminance $(L)$ measurements, emission spectra (also radiant intensity and radiance), and device efficiencies are less than 1,5 , and $10 \%$, respectively). The transmittance of the RSL was measured using a spectrophotometer (Hitachi U-3501). 


\section{Results and discussion}

Figure 1(a) shows the device structure of the TOLEDs under study. It is based on the $p-i-n$ structure for power efficient device operation, and the emission layer in the middle consists of double emission layers resulting in a broad emission zone and thus reduced roll-off [15]. The sky-blue organic emitter FIrpic with a peak emission wavelength at $475 \mathrm{~nm}$ was used as its emission is absorbed better by DCM molecules (absorption maximum at $480 \mathrm{~nm}$ ) than the emission from fluorescent blue emitters [16]. In addition, it using phosphorescence from triplet excitons also improves device efficiency [17]. DCM-doped Alq3 (2 wt\%) layers with various thicknesses were deposited on the top of the Ag cathode to perform color-conversion and create white emission in the top direction $[16,18,19]$. Since the color-conversion layer also forms an outermost dielectric capping layer, its thickness was optimized with a custommade optical simulation tool considering dipole emission and Purcell effect [20] with results shown in Fig. 1(b). The external quantum efficiency (EQE) shown on the y-axis of the figure was calculated assuming $100 \%$ intrinsic radiative quantum yield $(q)$ and an electrical efficiency $(\gamma)$ close to unity [20]. As the capping layer thickness varies, reflectance intensity/phase at the top electrode also changes and so does the overall device efficiency. According to the simulation, the total sum of the bottom/top direction photon counts (i.e. total $\mathrm{EQE})$ reaches local maxima at capping layer thickness values of $100 \mathrm{~nm}, 265 \mathrm{~nm}, 435 \mathrm{~nm}$, $550 \mathrm{~nm}$ and so on. Although the absolute maximum in OLED efficiency occurs at a capping thickness of $100 \mathrm{~nm}$, such a thin color-conversion layer will not provide sufficient conversion length to realize white light. We have therefore excluded the $100 \mathrm{~nm}$ case. As we also found that thicknesses beyond $500 \mathrm{~nm}$ significantly deteriorate device efficiency, we adopted two thickness values of $265 \mathrm{~nm}$ and $435 \mathrm{~nm}$. Even though this optimization of capping layer thickness was performed assuming zero down-conversion (which is essentially a macroscopic randomizing process that is difficult to be taken into consideration in our calculation model), 
we believe that it still provides a valid estimate of bi-directional total flux of our TOLEDs since (i) unconverted bottom emission is still dominant as can be seen in Fig. 1(c), and (ii) ratio between unconverted/converted photons in top emission is also high, as will be described later. Because the capping layer adjustment only affects the optical characteristics of the TOLEDs but not the electrical properties of the device, only the $L-V$ curves show noticeable differences between devices while the $J-V$ are identical within the margin of error as shown in Fig. 1(c).

To further understand the effect of the color-conversion capping layer on device performance, electroluminescence (EL) spectra in forward direction were recorded for all three devices under study (capped with $0 \mathrm{~nm} / 265 \mathrm{~nm} / 435 \mathrm{~nm}$ ) and for both bottom/top directions. These are shown in Fig. 2(a) and (b) together with pictures of the operating devices. Bottom side EL spectra of the capped TOLEDs are similar to the uncapped TOLED with a slightly increased second shoulder peak of FIrpic at $500 \mathrm{~nm}$ indicating weak cavity effects in the devices. In contrast, top side EL spectra of the capped/uncapped TOLEDs exhibit a clear difference: The capping layer gives rise to another emission peak at a wavelength around $605 \mathrm{~nm}$ in the capped TOLED, creating white emission as shown in Fig. 2(b). Commission Internationale de l'Eclairage (CIE) 1931 color coordinates of the TOLEDs gradually shift from $(0.164,0.386)$ sky-blue color for the uncapped device to $(0.328,0.375)$ cold white color $(6,000 \mathrm{~K})$ for the device with $435 \mathrm{~nm}$ thick capping layer.

The device performance including current efficacy (CE) and EQE of the devices are summarized in Fig. 3(a)-(d). At a driving current density of $1 \mathrm{~mA} \mathrm{~cm}^{-2}$, the TOLEDs under study achieved a bottom side $\mathrm{CE}$ and EQE of $13.6 \mathrm{~cd} \mathrm{~A} \mathrm{~A}^{-1} / 15.7 \mathrm{~cd} \mathrm{~A}^{-1} / 15.8 \mathrm{~cd} \mathrm{~A}^{-1}$ and $5.6 \% / 6.5 \% / 6.3 \%$ (for $0 \mathrm{~nm} / 265 \mathrm{~nm} / 435 \mathrm{~nm}$ capping layer thicknesses), respectively. Compared to the uncapped TOLED, the 265 nm-capped devices shows a $\sim 16 \% \mathrm{EQE}$ enhancement. Another noticeable consequence is that even device efficiencies in just the top 
direction are further increased by applying the capping layer. CE and EQE values which are $2.8 \mathrm{~cd} \mathrm{~A}^{-1}$ and $1.2 \%$, respectively, for the top side of the uncapped TOLED are increased to $3.4 \mathrm{~cd} \mathrm{~A}^{-1}$ and $1.6 \%$ for the device with $265 \mathrm{~nm}$ capping layer (at a current density of $1 \mathrm{~mA}$ $\left.\mathrm{cm}^{-2}\right)-21 \%$ and $33 \%$ improvement in terms of CE and EQE. The fact that the EQE increase is larger than the $\mathrm{CE}$ enhancement may be due to the rearrangement of light propagation directions caused by absorption of blue photons and re-emission of orange-red photons by DCM molecules, similar to mechanisms reported in the literatures [21-23]. This demonstrates that color-conversion is feasible without compromising the device efficiency. Increasing color-conversion layer thickness from $265 \mathrm{~nm}$ to $435 \mathrm{~nm}$ further increases this rearrangement effect. The thicker capping layer also leads to slight decreased device efficiency presumably due to increased absorption losses and the limited conversion efficiency of the capping layers. However, even with the thickest conversion layer of $435 \mathrm{~nm}$, the capped TOLED exhibits EQE values higher than the uncapped device for a wide range of current density, demonstrating the usefulness of our approach.

CIE color coordinates of the top emission of the TOLEDs under study is shown in $10^{\circ}$ steps of viewing angles in Fig. 4(a). The $y$-coordinate of the top blue emission from the uncapped TOLED varies from $0.381\left(0^{\circ}\right.$, at the substrate normal) to 0.335 (at $80^{\circ}$ off-normal), owing to the cavity wavelength shift [24]. For top white emission from the TOLEDs, CIE coordinates range from $(0.302,0.389)$ at $0^{\circ}$ to $(0.256,0.364)$ at $80^{\circ}$ for the $265 \mathrm{~nm}$-capped device and from $(0.333,0.366)$ at $0^{\circ}$ to $(0.300,0.356)$ at $80^{\circ}$ for the $435 \mathrm{~nm}$-capped device. This corresponds to a relatively 'cold white' color with a correlated color temperature around 6,000-7,000K. We also measured the EL spectra and CIE coordinates in top direction at several representative forward luminance levels $\left(100,500\right.$ and $\left.1,000 \mathrm{~cd} \mathrm{~m}^{-2}\right)$ for the $265 \mathrm{~nm}$ capped TOLED as shown in Fig. 4(b). Differences were negligible $[\Delta(x, y)$ of $(0.002,0.002)]$ due to the intrinsic color stability advantage of the combination of a single-emitter OLED and 
a color-conversion layer.. In addition, to verify whether the color-conversion organic capping layer has no negative impact on the transparency of the TOLEDs, overall transmittance values of the uncapped/265 nm/435 nm-capped TOLEDs were measured in the visible wavelength range and are shown in Fig. 5.

While the uncapped TOLED exhibits an average transmittance of 33.9\%, $265 \mathrm{~nm}$ colorconversion organic layer slightly enhanced the average transmittance of the capped TOLED to be $34.9 \%$. Hence, the thin layer of DCM-doped Alq3 functions as an excellent nonscattering, non-diffusive color-conversion layer, while preserving the visual clarity of the TOLEDs by complementing the absorption loss with the optical capping effect. However, the TOLED with the $435 \mathrm{~nm}$ thick capping layer shows a reduced average transmittance of $30.0 \%$, consistent with the absorbing/directional redistributing nature of the DCM-doped $\mathrm{Alq}_{3}$ layer. This shows that careful control of the capping layer thickness can lead to both high efficacy and excellent transparency if a trade-off is obtained between the following three factors: 1) sufficient conversion length for down-conversion to white color, 2) optimal optical capping for maximized overall device efficiency, 3) restrained absorption from the capping layer to maintain the device transmittance.

\section{Conclusions}

To sum up, we have proposed a new type of transparent OLED that emits distinct colors into top and bottom direction. While the bottom-side emission exhibits blue light by the emission from FIrpic molecules, the DCM-doped $\mathrm{Alq}_{3}$ organic capping layer placed on top of the Ag top-electrode absorbs a portion of the blue photons and converts them into orange-red photons to create mixed white emission on the top side. The organic color-conversion capping layer plays three roles simultaneously in our color-asymmetric TOLEDs: as a colorconversion layer for the primary blue emission; as an outermost dielectric layer that alters the transmittance and reflectance at the top electrode to boost efficiencies in both top/bottom 
directions; and finally as an optical capping layer to maintain device transparency even with the absorption occurring within it. Our best bi-directional, color-asymmetric TOLED exhibited current efficacy (CE) and external quantum efficiency (EQE) of $15.7 \mathrm{~cd} \mathrm{~A}^{-1}$ and $6.5 \%$, respectively, in the blue bottom side emission and $3.4 \mathrm{~cd} \mathrm{~A}^{-1}$ and $1.6 \%$, respectively, in the cold white top side emission at a current density of $1 \mathrm{~mA} \mathrm{~cm}^{-2}$, while keeping the average device transmittancee in the visible range of $400 \mathrm{~nm}-750 \mathrm{~nm}$ at $34.9 \%$. Considering the unique visual behavior, this new type of TOLED is expected to turn into a whole new category of devices for OLED lighting.

\section{Acknowledgement:}

The authors thank Novaled AG, Dresden for cooperation. This work was in part financed by the European Social Fund and the Free State of Saxony through the OrthoPhoto project. J. Lee acknowledges the Alexander von Humboldt Foundation and the Industrial Strategic Technology Development Program (10045269, Development of Soluble TFT and Pixel Formation Materials/Process Technologies for AMOLED TV) funded by MOTIE/KEIT. S. Yoo acknowledges a financial support by the National Research Foundation of Korea Grant funded by the Korean Government (NRF-2011-013-D00046 and CAFDC/Seunghyup Yoo/No. 2013042126). 


\section{References}

[1] Z. B. Wang, M. G. Helander, J. Qiu, D. P. Puzzo, M. T. Greiner, Z. M. Hudson, S. Wang, Z. W. Liu, and Z. H. Lu, Nat. Photon. 5, 753 (2011).

[2] C. C. Wu, S. D. Theiss, G. Gu, M. H. Lu, J. C. Sturm, S. Wagner, and S. R. Forrest, IEEE Electron Device Lett. 18, 609 (1997).

[3] H. Cho, C. Yun, J. -W. Park, and S. Yoo, Org. Electron. 10, 1163 (2009).

[4] T. -H. Han, Y. Lee, M. -R. Choi, S. -H. Woo, S. -H. Bae, B. H. Hong, J. -H. Ahn, and T. -W. Lee, Nat. Photon. 6, 105 (2012).

[5] P. E. Burrows, S. R. Forrest, S. P. Sibley, and M. E. Thompson, Appl. Phys. Lett. 69, 2959 (1996).

[6] Z. Shen, P. E. Burrows, V. Bulovic, S. R. Forrest, and M. E Thompson, Science, 276, 2009 (1997).

[7] J. -S. Park, T. -W. Kim, D. Stryakhilev, J. -S. Lee, S. -G. An, Y. -S. Pyo, D. -B. Lee, and H. K. Chung, Appl. Phys. Lett. 95, 013503 (2009).

[8] J. Lee, S. Hofmann, M. Furno, M. Thomschke, Y.H. Kim, B. Lüssem, and K. Leo, Org. Electron. 12, 1383 (2011).

[9] G. Parthasarathy, C. Adachi, P. E. Burrows, and S. R. Forrest, Appl. Phys. Lett. 76, 2128 (2000).

[10] J. Meyer, T. Winkler, S. Hamwi, S. Schmale, H. -H. Johannes, T. Weimann, P. Hinze, W. Kowalsky, and T. Riedl, Adv. Mater. 20, 3839 (2008).

[11] A. R. Duggal, J. J. Shiang, C. M. Heller, and D. F. Foust, Appl. Phys. Lett. 80, 3470 (2002).

[12] J. Lee, N. Chopra, D. Bera, S. Maslov, S. -H. Eom, Y. Zheng, P. Holloway, J. Xue, and F. So, Adv. Energy Mater. 1, 174 (2011).

[13] T. -W. Koh, H. Cho, C. Yun, and S. Yoo, Org. Electron. 13, 3145 (2012). 
[14] J. -I. Lee, J. Lee, J. -W. Lee, D. -H. Cho, J. -W. Shin, J. -H. Han, and H. Y. Chu, ETRI. J. 34, 690 (2012).

[15] S. -J. Su, E. Gonmori, H. Sasabe, and J. Kido, Adv. Mater. 20, 4189 (2008).

[16] V. Gohri, S. Hofmann, S. Reineke, T. Rosenow, M. Thomschke, M. Levichkova, B. Lüssem, and K. Leo, Org. Electron. 12, 2126 (2011).

[17] S. -J. Yeh, M. -F. Wu, C. -T. Chen, Y. -H. Song, Y. Chi, M. -H. Ho, S. -F. Hsu, and C. H. Chen, Adv. Mater. 17, 285 (2005).

[18] S. Chen, and H. -S. Kwok, Org. Electron. 12, 677 (2011).

[19] Y. B. Yuan, S. Li, Z. Wang, H. T. Xu, and X. Zhou, Opt. Express 17, 1577 (2009).

[20] M. Furno, R. Meerheim, S. Hofmann, B. Lüssem, and K. Leo, Physical Review B, 85, 115205 (2012).

[21] D. Bera, S. Maslov, J. Lee, J. Xue, F. So, and P. H. Holloway, J. Photon. Energy 1, 011025 (2011).

[22] A. Kumar, R. Srivastava, P. Tyagi, D. S. Mehta, and M. N. Kamalasanan, Synthetic Met. 161, 1172 (2011).

[23] B. C. Krummacher, M. Mathai, F. So, S. Choulis, and V. E. Choong, J. Display Technol. 3, 200 (2007).

[24] A. Dodabalapur, L. J. Rothberg, R. H. Jordan, T. M. Miller, R. E. Slusher, and J. M. Philips, J. Appl. Phys. 80, 6954 (1996). 


\section{Figure Captions}

\section{Figure 1}

(a) Device structure of the proposed TOLEDs (b) Result of optical simulation of the external quantum efficiency of the TOLED versus capping layer thickness (c) $J-V-L$ graphs of the devices under study

\section{Figure 2}

Electroluminescence (EL) spectra of the devices with various capping layer thicknesses measured in the forward direction and pictures of operating devices, (a) at bottom side and (b) top side. EL spectra were obtained at a driving current of $3 \mathrm{~mA}$ and photos were taken at a driving current of $1 \mathrm{~mA}$.

\section{Figure 3}

Current efficiency (CE) measured normal to the substrate (a) at bottom side (b) at top side, and external quantum efficiency (EQE) measured at (c) bottom side and (d) top side versus current density. Enhancement in $\mathrm{EQE}$ is larger than in $\mathrm{CE}$, indicating that the color conversion capping layer redistributes light propagation direction within TOLEDs.

\section{Figure 4}

(a) Shifts in CIE $1931 x y$-coordinates for the top side emission of three TOLEDs according to the viewing angle changes, measured from $0^{\circ}$ (direction normal to the substrate) to $80^{\circ}$. (b) EL spectra and CIE coordinates of the 265-nm capped TOLED at various luminance values in the top side emission, demonstrating an exceptional color stability.

\section{Figure 5}

Transmittance of the TOLEDs under study measured in the visible wavelength range. The average transmittance is $33.9 \% / 34.9 \% / 30.0 \%$ for the $0 \mathrm{~nm} / 265 \mathrm{~nm} / 435 \mathrm{~nm}$-capped device, respectively, which is appropriate for see-through applications. 
(a)
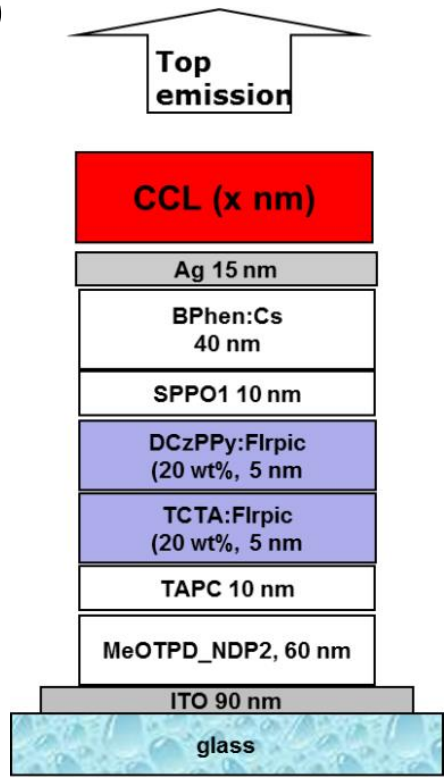

Bottom emissio (b)

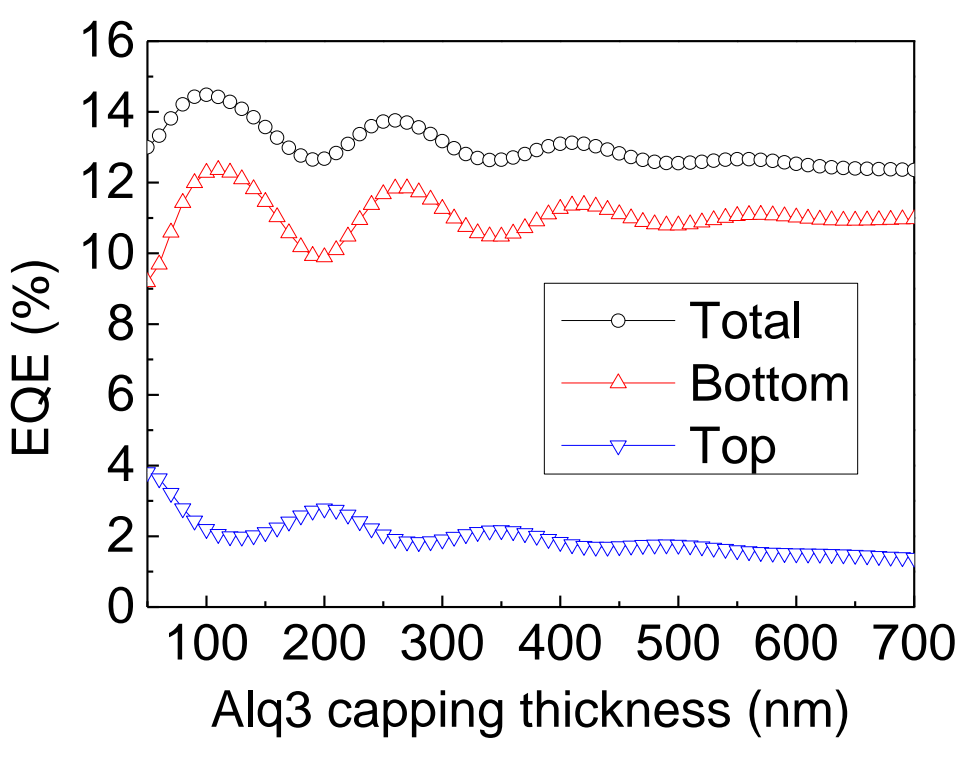

(c)

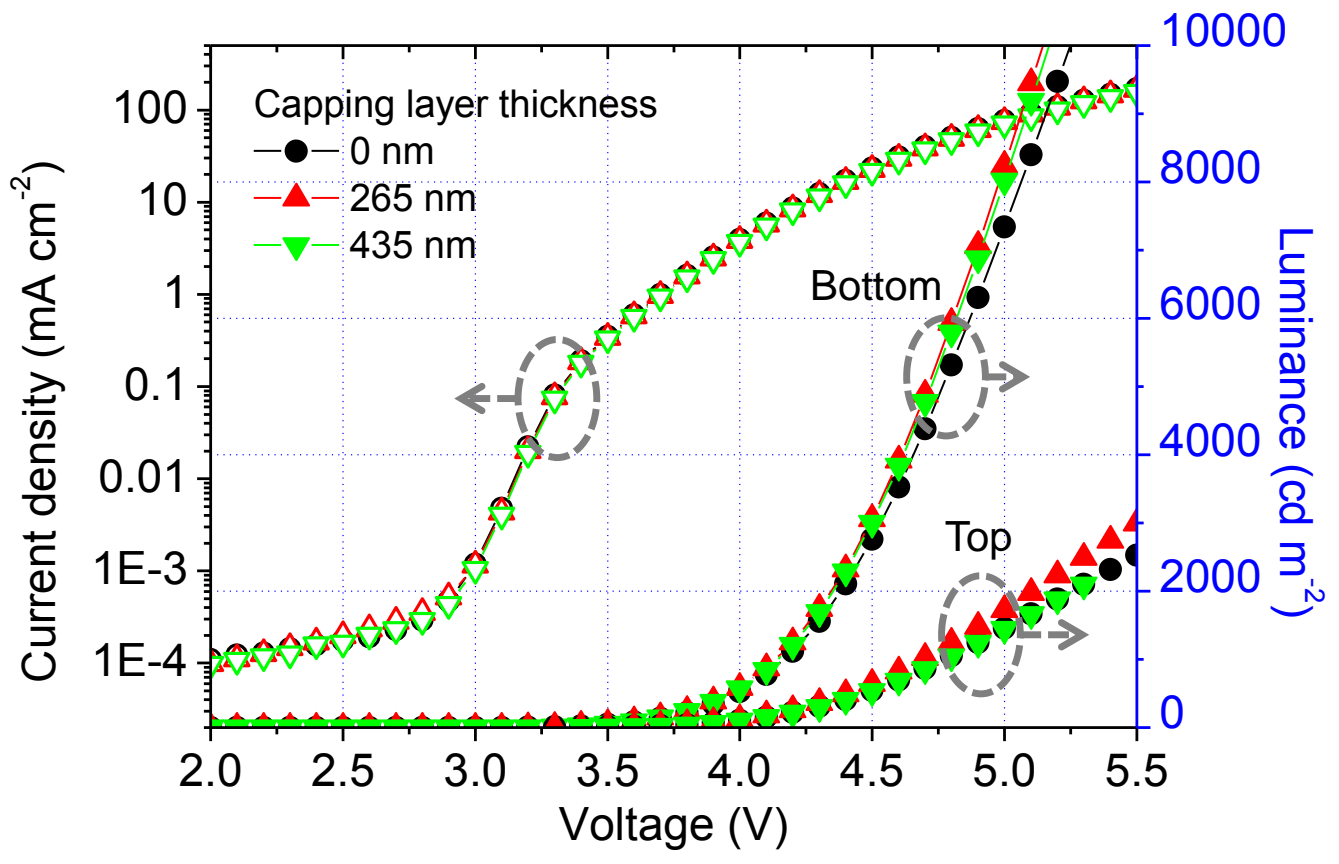


(a)
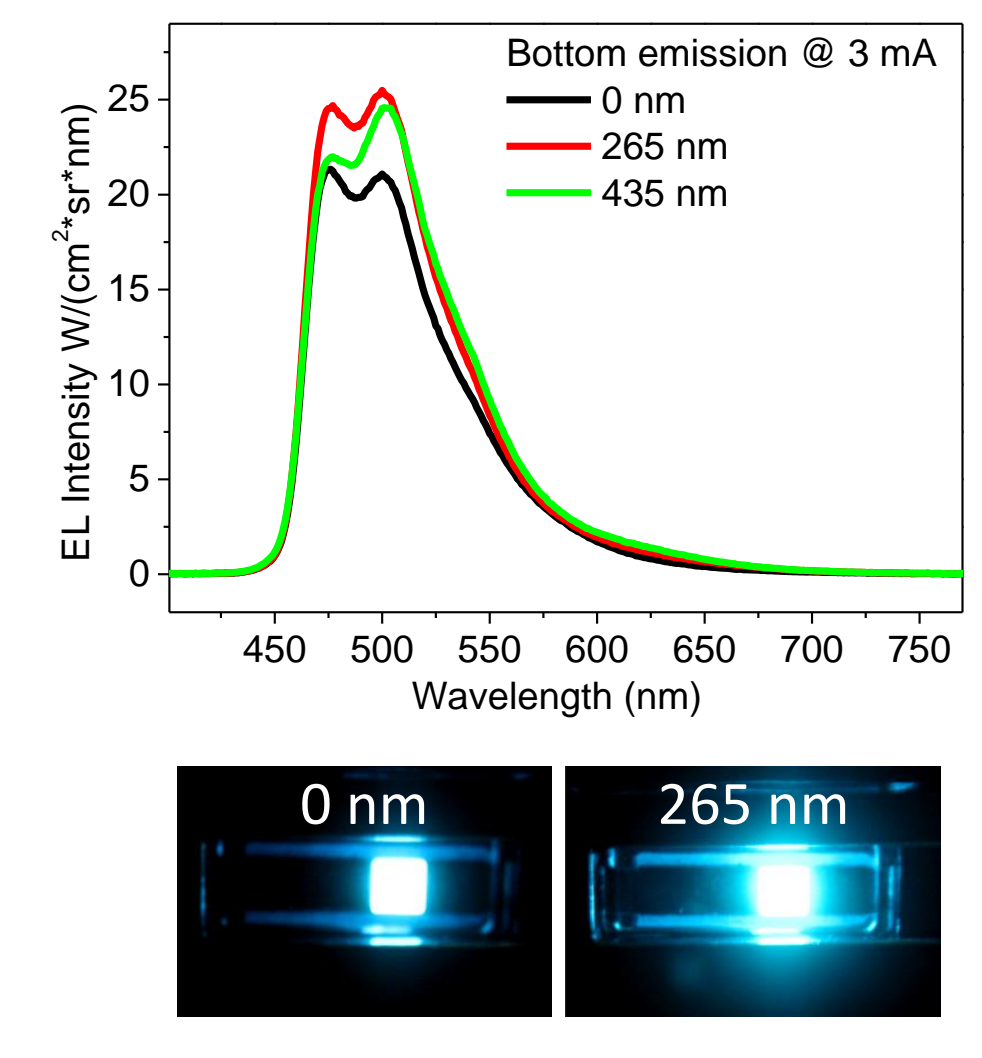

(b)
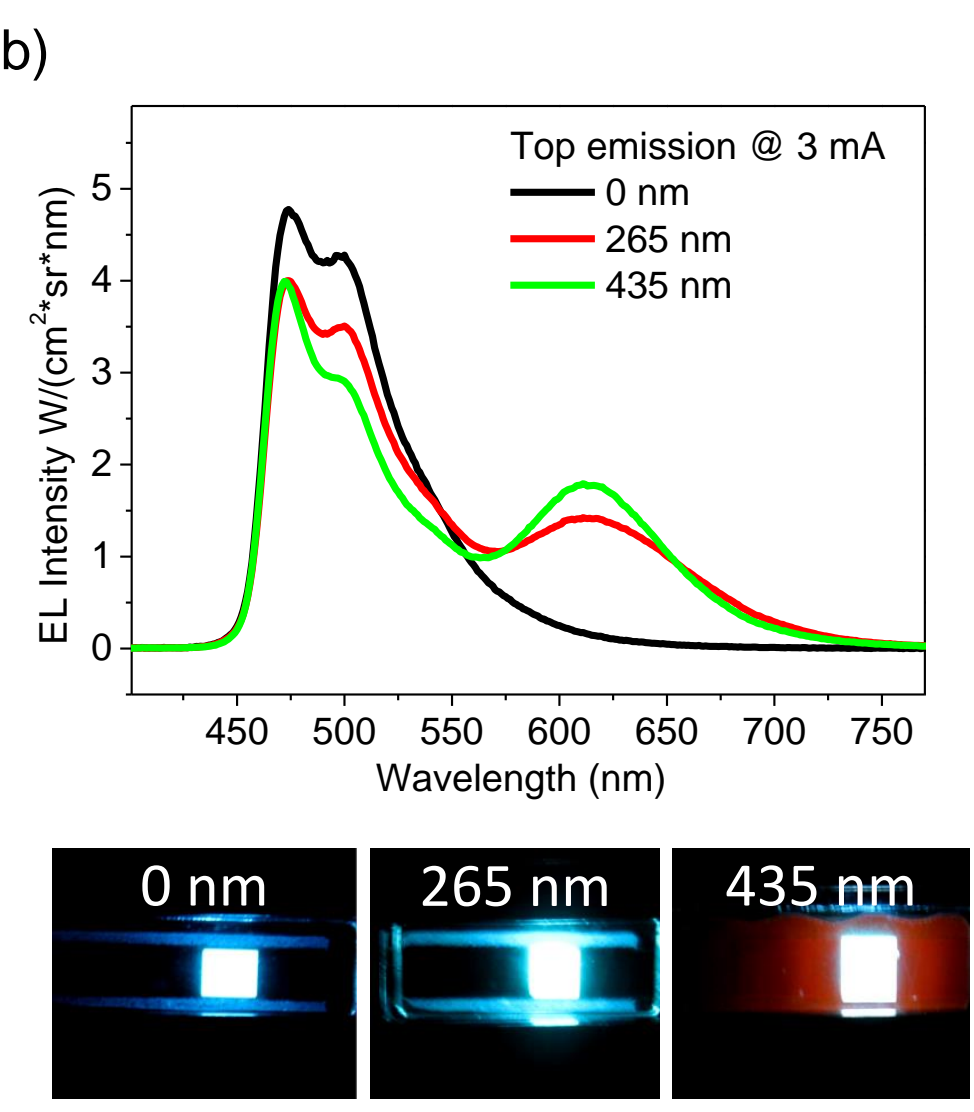

$435 \mathrm{~nm}$

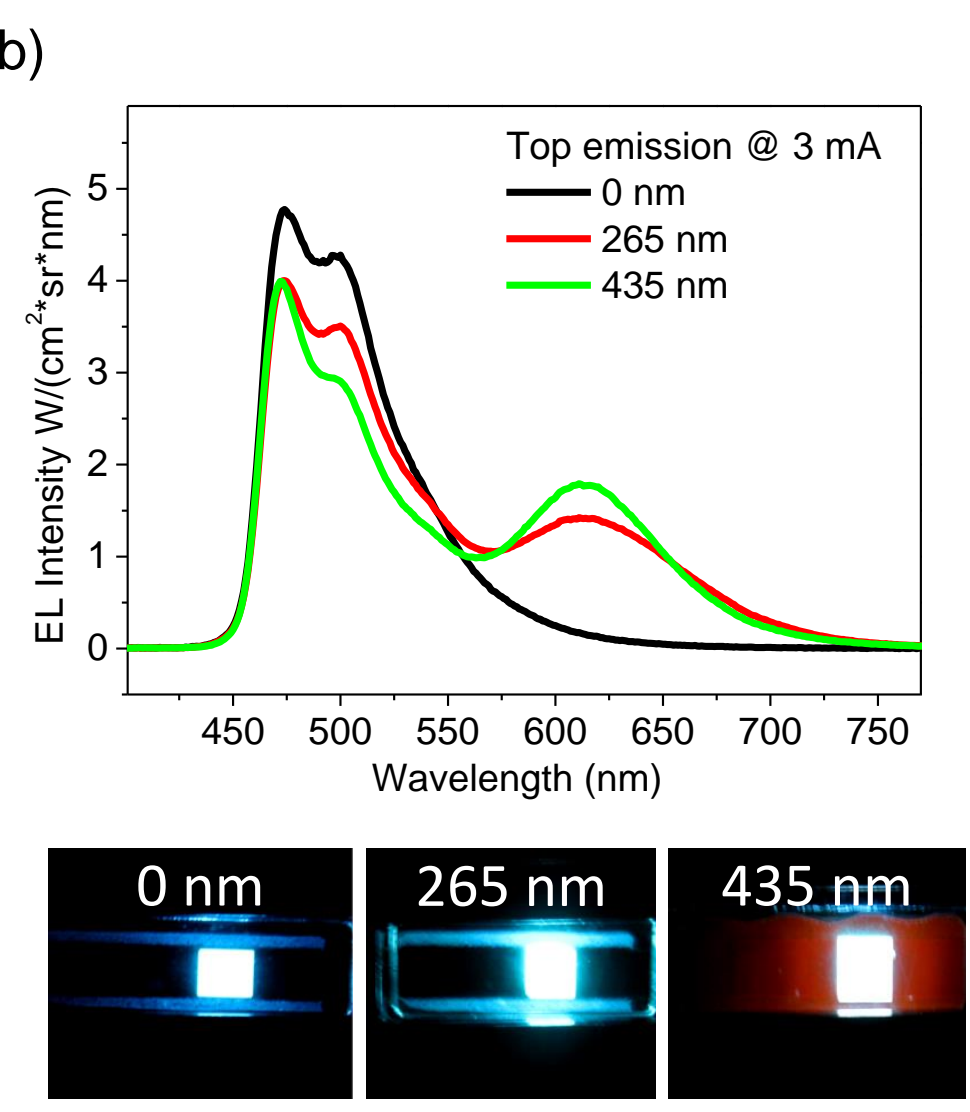

.
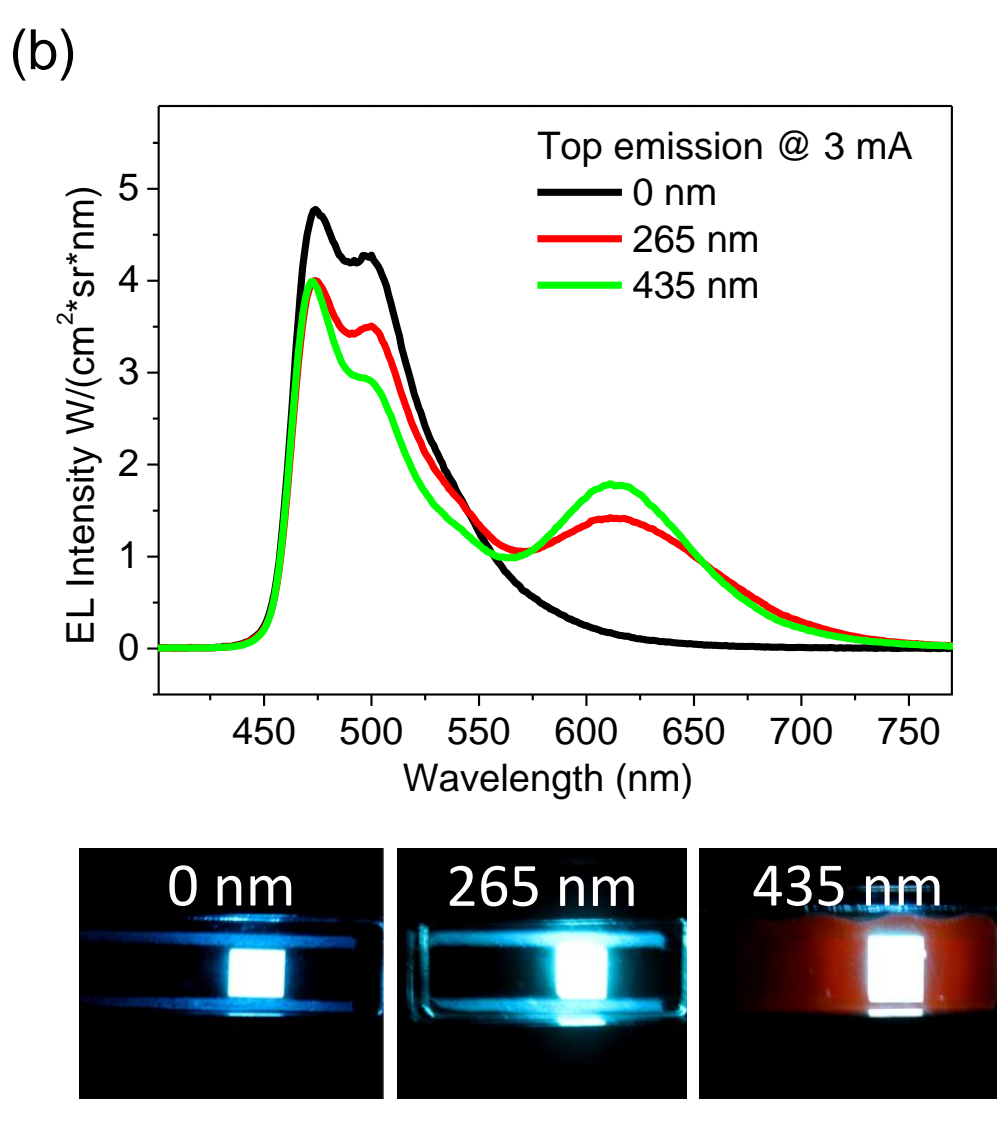
(a)

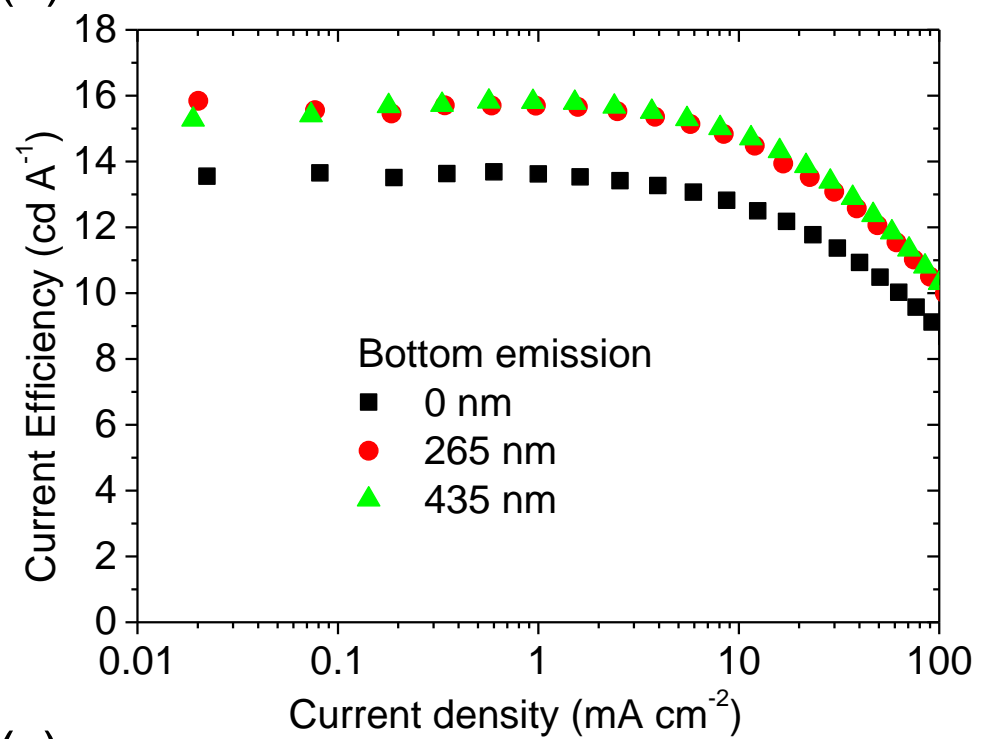

(c)

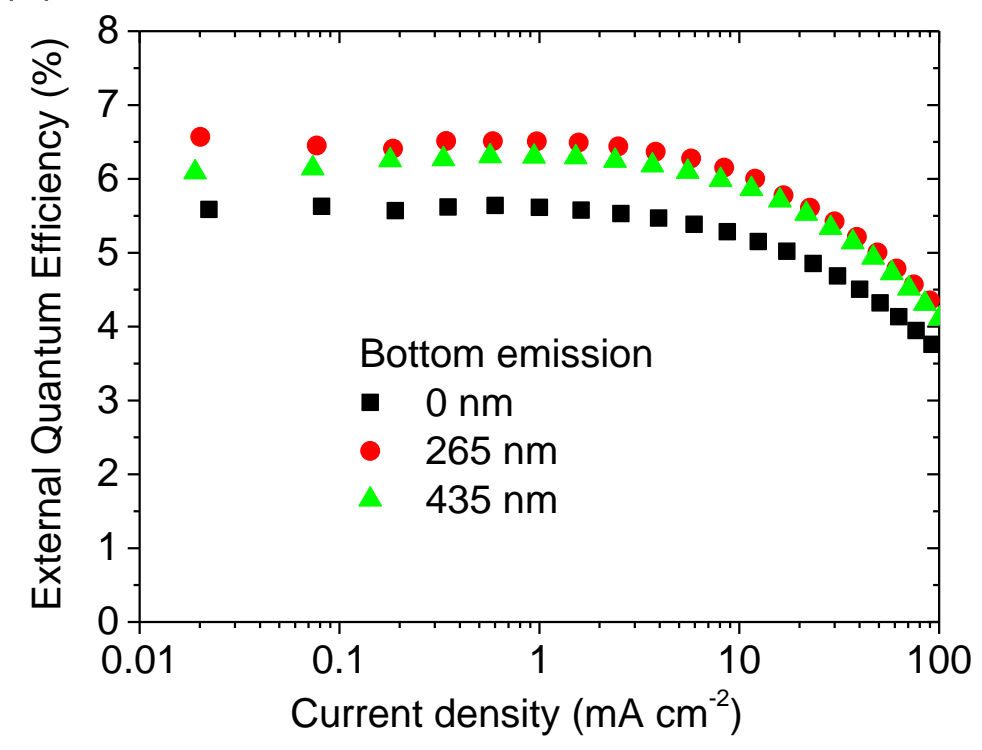

(b)

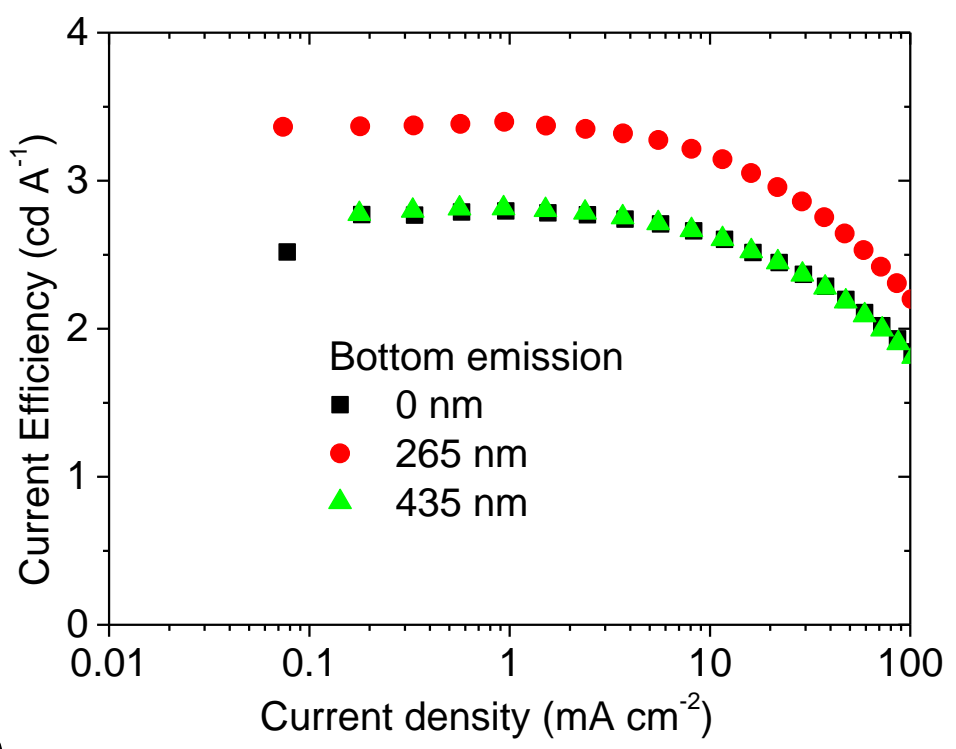

(d)

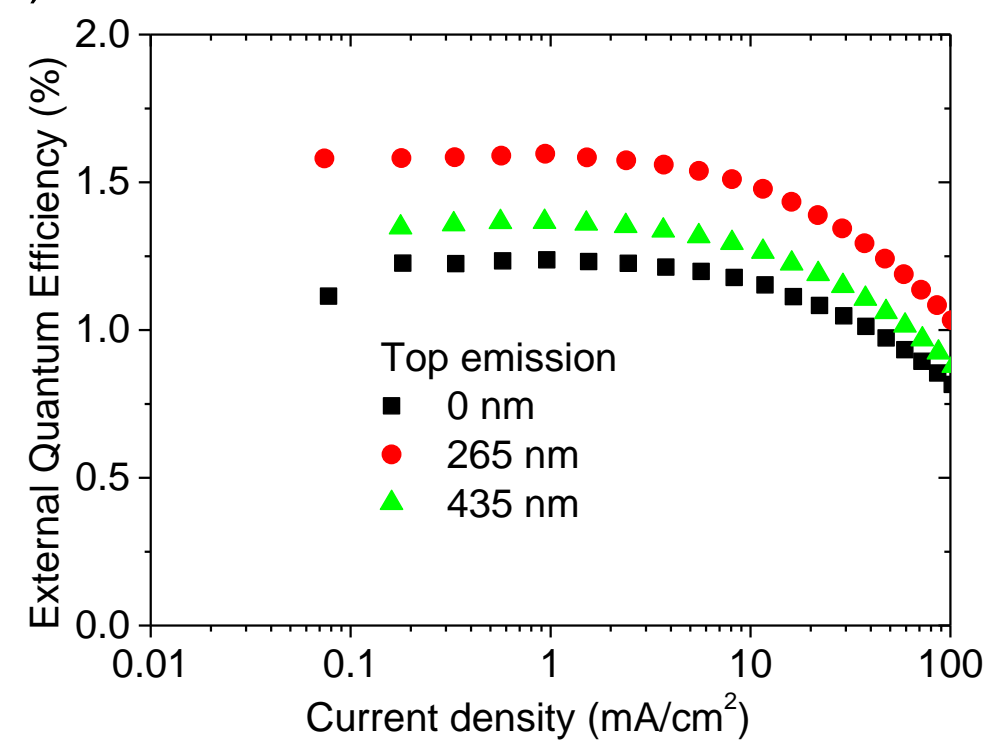


(a)
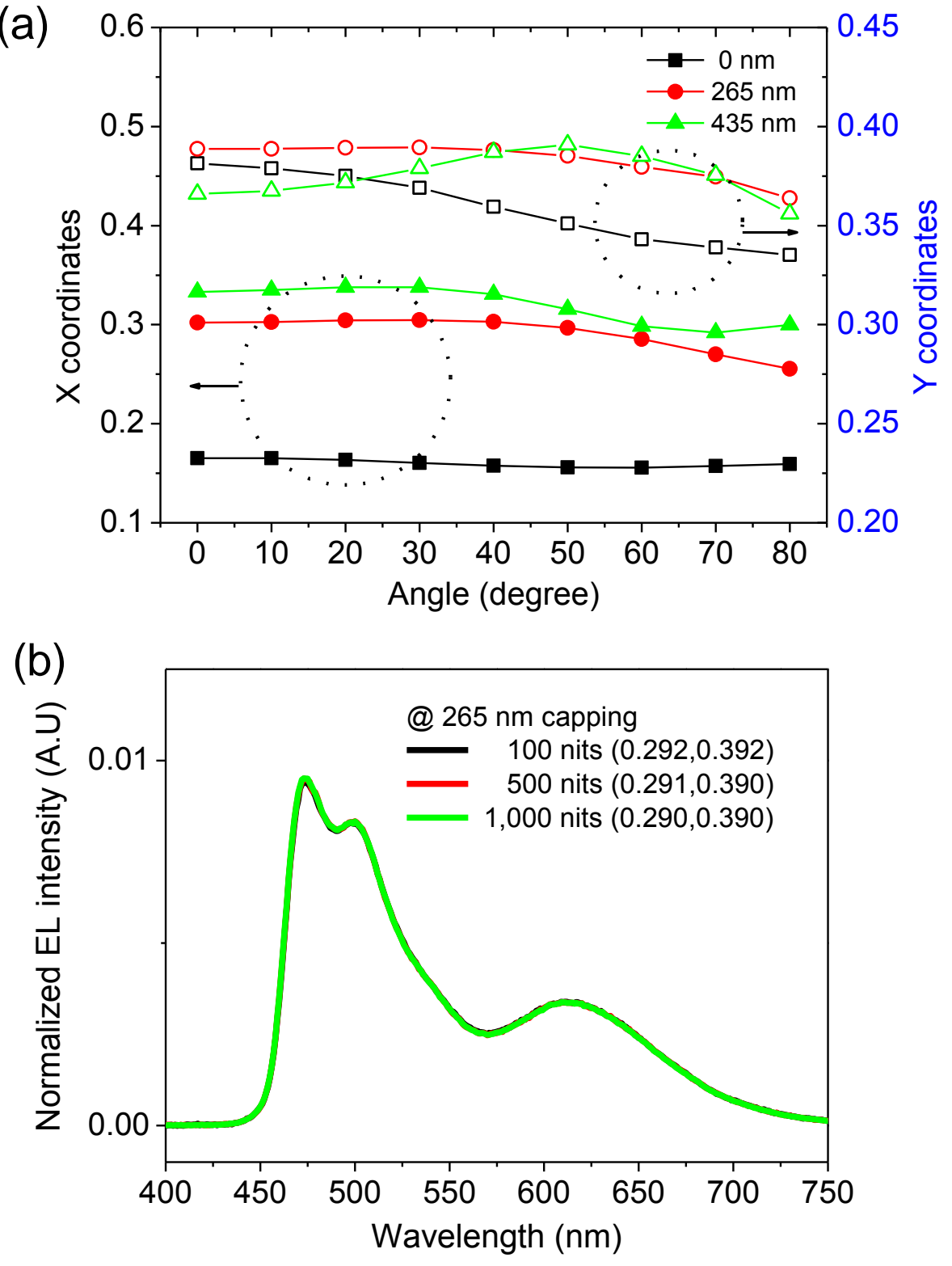


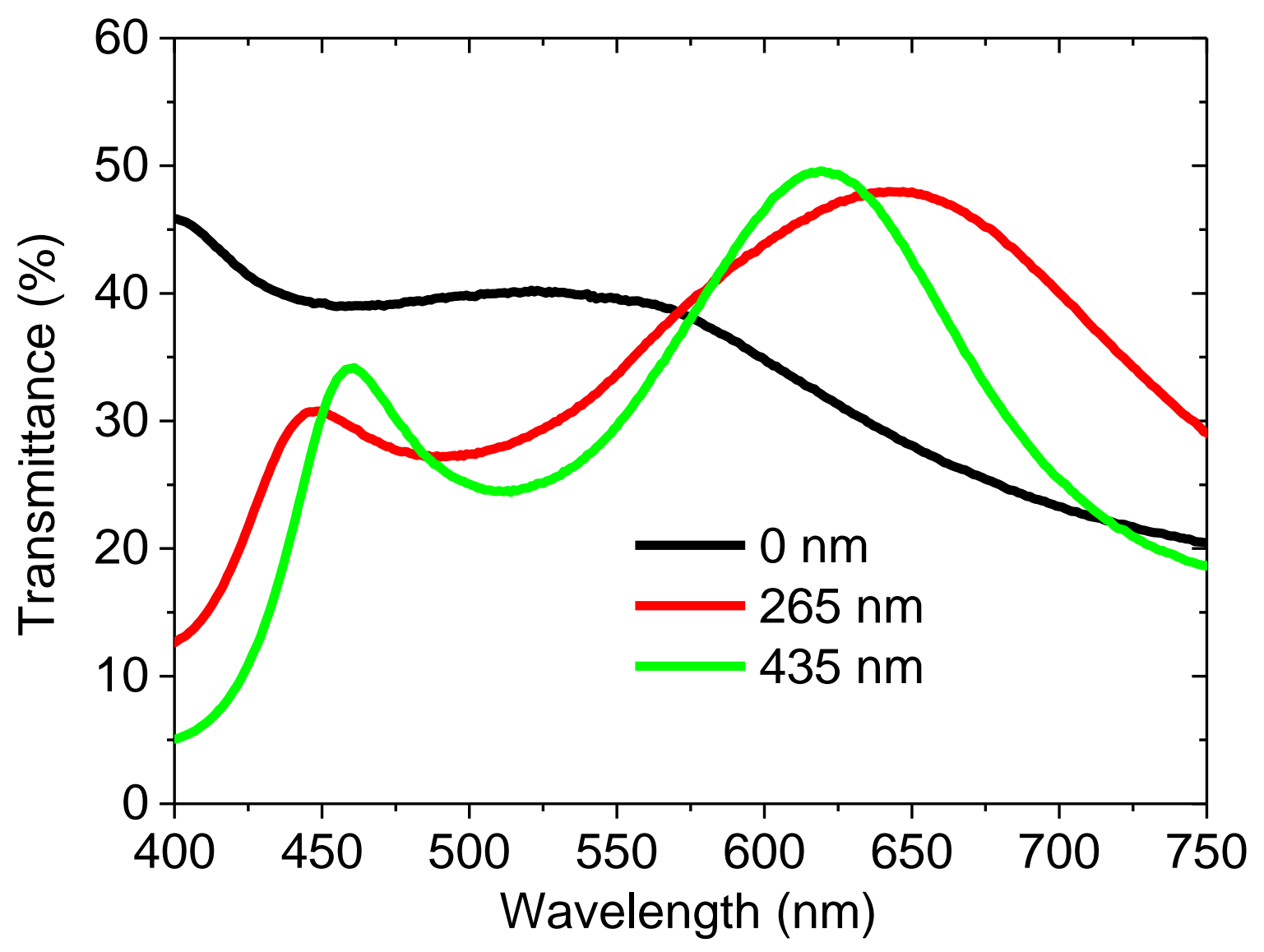

